\title{
The scoring systems in predicting short-term outcomes in patients with hepatitis B virus-related acute-on-chronic liver failure
}

\author{
Hui Li ${ }^{1,2 \#}$, Jun Zheng ${ }^{1 \#}$, Liang Chen ${ }^{1 \#}$, Jianye Cai ${ }^{1}$, Min Zhang $^{3}$, Genshu Wang ${ }^{1}$ \\ ${ }^{1}$ Department of Hepatic Surgery and Liver Transplantation Center, the Third Affiliated Hospital of Sun Yat-sen University, Guangzhou, China; \\ ${ }^{2}$ Department of Liver Surgery, West China Hospital, Sichuan University, Chengdu, China; ${ }^{3}$ Department of Anesthesiology, Jiulongpo People's \\ Hospital, Chongqing, China \\ Contributions: (I) Conception and design: G Wang, H Li, M Zhang; (II) Administrative support: G Wang; (III) Provision of study materials or \\ patients: G Wang, J Zheng; (IV) Collection and assembly of data: H Li, J Zheng, L Chen; (V) Data analysis and interpretation: H Li, J Zheng, L \\ Chen, J Cai; (VI) Manuscript writing: All authors; (VII) Final approval of manuscript: All authors. \\ "These authors contribute equally to this work. \\ Correspondence to: Genshu Wang. Department of Hepatic Surgery and Liver Transplantation Center, the Third Affiliated Hospital of Sun Yat-sen \\ University, Guangzhou, China. Email: wgsh168@163.com; Min Zhang. Department of Anesthesiology, Jiulongpo People’s Hospital, Chongqing, \\ China. Email: zhm03030802@163.com.
}

Background: Hepatitis B virus related acute-on-chronic liver failure (HBV-ACLF) is a life-threatening syndrome characterized by acute and severe hepatic insults with high short-term mortality. This study aimed to compare the scoring systems which were used to predict short-term outcomes for HBV-ACLF patients.

Methods: A total of 529 patients diagnosed as HBV-ACLF were retrospectively analyzed and randomly divided, at a ratio of $3: 1$, into derivation cohort $(n=397)$ and validation cohort $(n=132)$. Univariate and multivariate analyses were performed to determine the discriminative abilities of the ALBI grade in predicting 30-day and 90-day mortality. The area under the receiver operating characteristic (AUC) curves was used to evaluate the accuracy of models.

Results: The survival was associated with lower ALBI score, MELD score and CLIF-C ACLF score than death. In the derivation cohort, elevated ALBI score was related to worse prognosis (30-day mortality: HR =3.452; 90-day mortality: HR =3.822), increased MELD score was associated with worse overall survival (30day mortality: HR =1.073; 90-day mortality: HR =1.082), and increased CLIF-C ACLF score was associated with worse overall survival (30-day mortality: $\mathrm{HR}=1.061$; 90-day mortality: $\mathrm{HR}=1.065$ ). The multivariate analyses identified the ALBI score, MELD score and CLIF-C ACLF score as independent prognostic predictors. The results of validation cohort validated these findings.

Conclusions: Our study revealed that both the ALBI score, MELD score and CLIF-C ACLF score could predict 30- and 90-day mortality of HBV-ACLF accurately. Elevated ALBI score, MELD score and CLIF-C ACLF score were associated with worse prognosis.

Keywords: Hepatitis B virus related acute-on-chronic liver failure (HBV-ACLF); ALBI score; MELD score; CLIF-C ACLF score

Submitted Mar 10, 2020. Accepted for publication Jul 14, 2020.

doi: 10.21037/apm-20-608

View this article at: http://dx.doi.org/10.21037/apm-20-608 


\section{Introduction}

Acute-on-chronic liver failure (ACLF) is a life-threatening syndrome that occurs in patients with either previously diagnosed or undiagnosed chronic liver disease $(1,2)$. It is characterized by acute and severe hepatic insults manifesting as coagulopathy and jaundice, complicated within four weeks by ascites and/or hepatic encephalopathy (3), and with high short-term mortality of 63-72.3\% (4-6). In Asia, especially in China, the leading cause for ACLF is hepatitis $B$ virus (HBV) infection with the proportion of more than $70 \%$ (7). Liver transplantation is still the only therapeutic strategy that has been verified to be beneficial for ACLF patients. However, the lack of available donors restricts its application (8). Therefore, it is critical to monitor the disease progression and distinguish ACLF patients that need emergency liver transplantation.

To predict the short-term outcomes accurately, several scoring systems such as Child-Pugh classification (CP), model of end-stage liver disease (MELD) (9), MELD sodium (MELD-Na) (10) were developed. Chronic liver failure-sequential organ failure assessment (CLIFSOFA) was developed to evaluate multiple organ failure initially, then modified as CLIF-CONSORTIUM score for ACLF patients (CLIF-C ACLFs). It had been proved the CLIF-C ACLFs was higher predictive accurate than CP, MELD and MELD-Na (11). The CP and MELD model are still routinely applied for allocation of donor livers due to their feasibility. The CP classification is based on five parameters including total serum bilirubin, serum albumin, prothrombin time, degree of ascites and extent of hepatic encephalopathy $(12,13)$. However, subjective clinical assessment of the extent of ascites and hepatic encephalopathy limits its clinical application (14).

The albumin-bilirubin (ALBI) grade was initially developed to assess the severity of liver dysfunction in patients with hepatocellular carcinoma (HCC) by Johnson and colleagues (15). It is calculated from serum albumin and bilirubin using the formula: ALBI score $=\left(\log _{10}\right.$ bilirubin $\times$ $0.66)-($ albumin $\times 0.085)$, where bilirubin is in $\mathrm{mol} / \mathrm{L}$ and albumin in $\mathrm{g} / \mathrm{L}$. The cut-off points were as follows: $\leq-2.60$ (ALBI grade 1 ), more than -2.60 to $\leq-1.39$ (ALBI grade 2 ), and more than -1.39 (ALBI grade 3 ). Plenty of studies have verified its accuracy in predicting prognosis of $\mathrm{HCC}$ patients (16-19). Moreover, Chan et al. demonstrated that the ALBI grade was an independent prognostic factor for patients with primary biliary cirrhosis and superior to $\mathrm{CP}$ classification and MELD (20). However, it remains unclear about the feasibility of the ALBI grade in predicting shortterm outcomes of patients with hepatitis $\mathrm{B}$ virus related ACLF (HBV-ACLF).

The current study aimed to compare the accuracy of the scoring systems in predicting short-term outcomes of $\mathrm{HBV}$-ACLF patients. We present the following article in accordance with the STROBE reporting checklist (available at http://dx.doi.org/10.21037/apm-20-608).

\section{Methods}

\section{Study population}

We retrospectively enrolled patients who were newly diagnosed as HBV-ACLF from June 2012 to July 2015 and admitted in the Third Affiliated Hospital of Sun Yat-sen University. ACLF was diagnosed according to the consensus recommendations of the Asian Pacific Association for the Study of the Liver (APASL), which included jaundice with a serum total bilirubin level of $\geq 5 \mathrm{mg} / \mathrm{dL}$, coagulopathy with an INR of $\geq 1.5$ or prothrombin activity less than $40 \%$ and development of ascites and/or encephalopathy as determined by physical examination within 4 weeks $(3,21)$. Patients with liver cancer and other malignancies were excluded. Patients were excluded if they were coinfected with human immunedeficiency virus (HIV), hepatitis A, C, D, or E virus, or accompanied by alcoholic hepatitis, drug-induced hepatitis, autoimmune hepatitis. The ACLF patients received nucleotide antiviral therapies and treatment strategies according to the diagnostic and treatment guidelines for ACLF adopted by the Chinese Medical Association (22). All eligible patients or their relative gave written informed consent. This study was approved by the ethics committee of the Third Affiliated Hospital of Sun Yat-sen University (the ethical number: 2014-142), in accordance with the guidelines of the Declaration of Helsinki (as revised in 2013) (23). The flow diagram was shown in Figure S1.

\section{Data collection}

The following data of pretreatment clinical information were reviewed and collected from the hospital electronic medical records: name, gender, age, hemoglobin (HGB), platelet count (PLT), normalized ratio (INR), serum alanine transaminase (ALT), aspartate transaminase (AST), albumin (ALB), total bilirubin (TBIL), $\gamma$-glutamyl transpeptidase (GGT), creatinine (Cr), blood urea nitrogen (BUN). All the 
included patients were followed up at least for 90 days.

Calculation formula for models were as following: MELD score $=9.6 \times \ln (\mathrm{Cr})+3.8 \times \ln (\mathrm{TBIL})+11.2 \times \ln (\mathrm{INR})+$ $6.4 \times$ (etiologies), where $\mathrm{Cr}$ is in $\mathrm{mg} / \mathrm{dL}$; ALBI score = $\left(\log _{10}\right.$ bilirubin $\left.\times 0.66\right)-($ albumin $\times 0.085)$, where bilirubin is in mol/L and albumin in $\mathrm{g} / \mathrm{L}$; the CLIF-C ACLF was calculated as previous described (11). The classification of the ALBI grade were: $\leq 2.60$ (ALBI grade 1), >-2.60, $\leq-1.39$ (ALBI grade 2), and >-1.39 (ALBI grade 3) (15).

\section{Statistical analysis}

All statistical analyses were performed using SPSS (version 22.0, Chicago, IL, USA), MedCalc (version 15.2.2.0, Ostend, Belgien) and Graphpad Prism (version 8.0, San Diego, California, USA). Student's $t$-test and Chi-square test were used to assess the correlation of continuous and categorical variables to relevant outcomes respectively. The most used cut-off value for ALBI score was used in this study (24). The optimal thresholds of MELD and CLIF-C ACLF score were identified by application of the receiver operating characteristics (ROC) curve using 90-day mortality as the discriminant (25). The Z-test was used to compare the area under the receiver operating characteristic (AUC). Kaplan-Meier method was used to perform survival analysis for the groups with different cut-off values, and their differences were tested with log-rank test. Subsequently, Cox proportional hazards regression model (enter method) was used to identify potential independent prognostic factors for 30- and 90-day mortality of HBVACLF patients. A two-tailed $\mathrm{P}$ value less than 0.05 was considered statistically significant.

\section{Results}

\section{Patients characteristics}

A total of 597 patients newly diagnosed with HBV-ACLF were admitted in the Third Affiliated Hospital of Sun Yat-sen University. Of them, 68 were excluded: 21 were associated with HCC; 8 were coinfected with hepatitis A, $\mathrm{C}$ or $\mathrm{E}$ virus; 13 were caused by alcoholic hepatitis; 26 were associated with severe extra-hepatic diseases (Figure S1). Finally, 529 patients were included in the present study. They were randomly divided into derivation and validation cohort at a ratio of $3: 1$. Therefore, 397 patients [334 (84.1\%) male; mean age, 45.09 years] were classified into derivation cohort, whereas 132 patients [58 (86.5\%) male; mean age,
43.76 years] were used to validate our findings. The baseline demographic and clinical characteristics were summarized in Table 1. The optimal cut-off values for MELD and CLIF-C ACLF were 27.83 and 48.63, respectively.

\section{Derivation cobort}

At the endpoint of 30-day after diagnosis, 291 (73.3\%) patients were survived. The survival showed a decreased ALBI score, MELD score and CLIF-C ACLF score than those of death (Figure $1 A, B, C)$. A total of $330(83.1 \%$ ) patients with liver function of ALBI grade 3 showed worse prognosis than those with ALBI grade 2 [hazard ratio (HR): 3.452; 95\% confidence interval (CI): 2.272-5.244]. A total of 135 (34.1\%) patients with high MELD score were associated with worse overall survival than those with low MELD score (HR: 1.073, 95\% CI: 1.043-1.105), whereas $111(27.9 \%)$ patients with increased CLIF-C ACLF score showed worse prognosis (HR: 1.061, 95\% CI: 1.039-1.085). The multivariate analysis identified the elevated ALBI score, MELD score and the CLIF-C ACLF score as independent risk factors in predicting 30-day mortality (Table 2). The ALBI score showed larger AUC than the MELD score and CLIF-C ACLF score (AUCs: 0.682 versus 0659, 0673) (Figure $S 2 A)$.

In total, $224(56.4 \%)$ patients were survived at 90day after diagnosed as HBV-ACLF. Similarly, the survival showed a decreased ALBI score, MELD score and CLIF-C ACLF score than those of death (Figure 1D,E,F). Patients with increased ALBI grade showed worse prognosis (HR: 3.822; 95\% CI: 2.505-5.832). Patients with high MELD score were associated with worse overall survival than those with low MELD score (HR: 1.082, 95\% CI: 1.056-1.108). Patients with increased CLIF-C ACLF score showed worse prognosis (HR: 1.065, 95\% CI: 1.047-1.084) (Figure 2). Among five parameters which were introduced in the multivariate analysis, the elevated ALBI score, MELD score and the CLIF-C ACLF score as independent risk factors in predicting 90-day mortality (Table 3). The AUCs for these three scoring systems were 0.67, 0.659 and 0.679, respectively (Figure $S 2 B$ ). The diagnostic accuracy of scoring systems for predicting 30 - and 90-day mortality was shown in Table S1.

\section{Validation cobort}

One hundred thirty two patients were included in 
Table 1 Baseline demographic and clinical characteristics of patients with HBV-ACLF in derivation cohort ( $\mathrm{n}=397)$ and validation cohort ( $\mathrm{n}=132$ )

\begin{tabular}{|c|c|c|}
\hline Variables & Derivation cohort $(n=397)$ & Validation cohort $(n=132)$ \\
\hline Male gender, n (\%) & $334(84.1 \%)$ & $58(86.5 \%)$ \\
\hline HGB (g/L) & $119.3 \pm 23.6$ & $119.1 \pm 23.7$ \\
\hline PLT $\left(10^{9} / \mathrm{L}\right)$ & $117.47 \pm 65.39$ & $121.86 \pm 78.949$ \\
\hline ALT (IU/L) & $650 \pm 813$ & $831 \pm 974$ \\
\hline AST (IU/L) & $462 \pm 677$ & $549 \pm 738$ \\
\hline ALB (g/L) & $31.48 \pm 4.68$ & $32.5 \pm 4.99$ \\
\hline TBIL ( $\mu \mathrm{mol} / \mathrm{L})$ & $373.73 \pm 167.56$ & $380.42 \pm 181.48$ \\
\hline BUN (mg/dL) & $4.88 \pm 4.69$ & $4.99 \pm 5.06$ \\
\hline ALBI score & $-1.02 \pm 0.39$ & $-1.09 \pm 0.38$ \\
\hline MELD score & $26.85 \pm 5.61$ & $26.6 \pm 5.47$ \\
\hline CLIF-CACLF score & $44.06 \pm 8.24$ & $43.27 \pm 7.73$ \\
\hline 30-day survival, n (\%) & $291(73.3 \%)$ & $98(74.2 \%)$ \\
\hline 90-day survival, n (\%) & $224(56.4 \%)$ & $78(59.1 \%)$ \\
\hline
\end{tabular}

Data were expressed as numbers of patients (\%) or mean \pm SD. HBV-ACLF, hepatitis B virus related acute-on-chronic liver failure; HGB, hemoglobin; PLT, platelet; INR, international normalized ratio; ALT, alanine aminotransferase; AST, aspartate aminotransferase; ALB, albumin; TBIL, total bilirubin; GGT, gamma glutamyl transpeptidase; Cr, creatinine; BUN, blood urea nitrogen; ALBI, albumin-bilirubin; MELD, model for end-stage liver diseases. CLIF-CACLF, CLIF-CONSORTIUM score for ACLF patients.

validation cohort. According to the cut-off values, 37 (28.0\%) were classified into ALBI grade 2 group, 93 (70.5\%) were classified into low MELD score group while $98(74.2 \%)$ were classified into low CLIF-C ACLF score group. Consistent to the derivation cohort, decreased ALBI grade, decreased MRLD score and CLIF-C ACLF score were associated with better prognosis (Figure 3). Among 15 clinicopathological characteristics, 8 were identified as potentially relevant with $\mathrm{P}$ values less than 0.05 in the univariate Cox regression analyses for 30-day mortality. Four of them were introduced in consequent multivariate analyses, the ALBI score, MELD score and CLIF-C ACLF score were identified as independent prognostic predictors for 30-day mortality (Table S2). Six clinicopathological characteristics were potential relevant in univariate analyses for 90-day mortality. Similarly, the ALBI score, MELD score and CLIF-C ACLF score were identified as independent prognostic predictors for 90-day mortality (Table S3).

\section{Discussion}

In the present study, we retrospectively analyzed 397 HBV-ACLF patients, and compared the accuracy of the ALBI score, MELD score and CLIF-C ACLF score. The results demonstrated that both ALBI score, MELD score and CLIF-C ACLF score were independent prognostic predictors for 30- and 90-day mortality in hospitalized HVB-ACLF patients receiving antiviral therapy and other clinical treatment. The initial findings were validated using an independent cohort. The ALBI score showed 

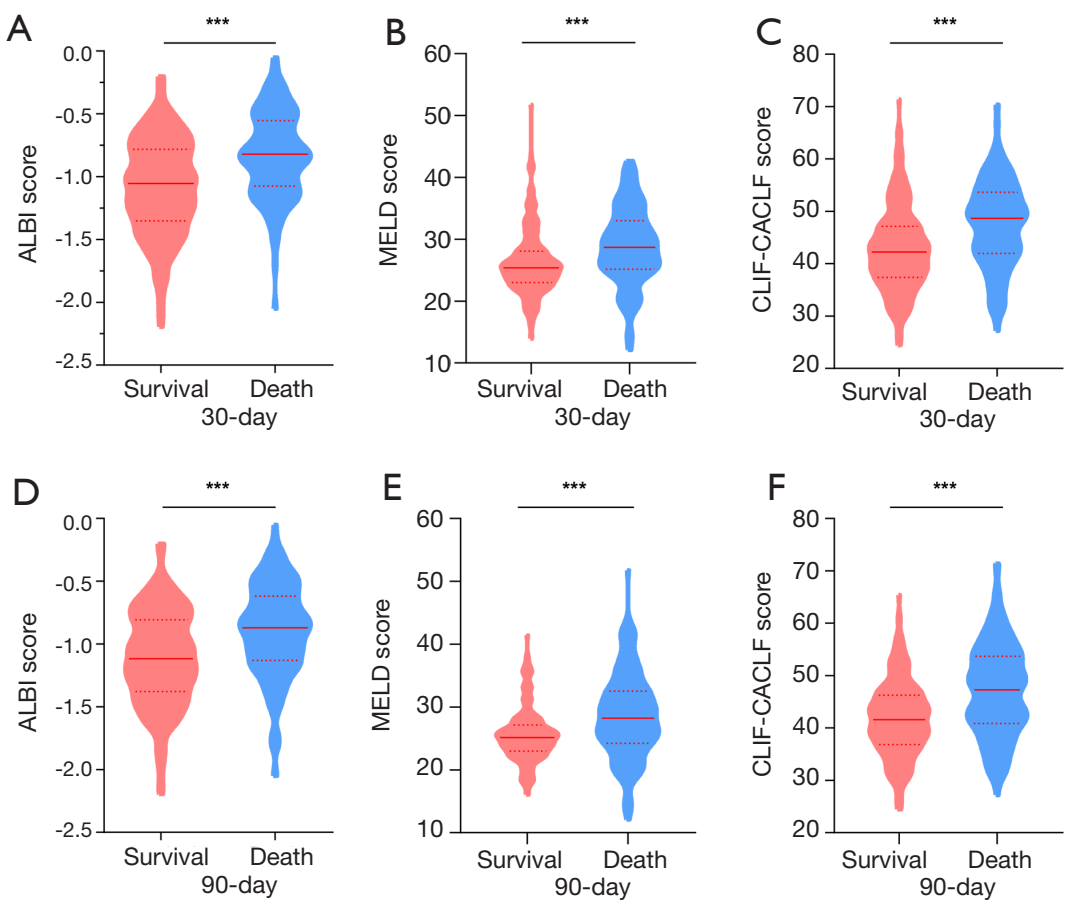

Figure 1 Violin plots showing the distribution of ALBI, MELD and CLIF-C ACLF score: in survival and death group at the end of 30-day (A, $\mathrm{B}$ and C), 90-day (D, E and F) after diagnosis. Solid lines represented median value, dotted lines represented quartiles. ${ }^{* * *}$, $<<0.001$. ALBI, albumin-bilirubin; MELD, model for end-stage liver diseases; CLIF-CACLF, CLIF-CONSORTIUM score for ACLF patients.

larger AUC than MELD score and CLIF-C ACLF score in predicting 30-day mortality. This would be helpful for clinical treatment strategies decision and selection of appropriate candidate for liver transplantation.

In China, HBV-ACLF accounts for almost $80 \%$ ACLF cases due to the high incidence of $\mathrm{HBV}$ infection, which would cause multiple organ failure severe function damage (26). Even antiviral therapy is a useful treatment strategy that would improve outcomes of HBV-ACLF patients (27), the mortality remains high. Emergency liver transplantation, the most effective therapeutic treatment, is limited by shortage of available donor livers. Therefore, it is important to lay emphasis on the allocation of donor livers. Accurate scoring systems which can predict prognostic outcomes are precondition for selection of candidates in the waiting list. Several scoring systems such as CP grade and MELD score have been developed to assess the severity of hepatic dysfunction and predict prognostic outcomes of HBV-ACLF. And they are commonly used for allocation of donor livers now. It is inspiring if any models show better accuracy of prognostic prediction than conventional scoring systems.

Among varies scoring systems for prognostic prediction of patients with HBV-ACLF, MELD score was one of the most widely used for organ allocation $(28,29)$. It involved three objective parameters, the total bilirubin, INR and creatinine. Recently, several scoring systems were reported to be superior to MELD score in accurate prognostic prediction of patients with ACLF. The CLIF-C ACLF, developed by Jalan et al., was demonstrated to be with better accuracy compared to MELD score, MELD-Na score (11). The acute physiology and chronic health evaluation (APACHE II) score showed higher predictive accuracy than MELD score, CP classification and SOFA (30). However, these models mostly analyzed patients in western countries with alcoholic hepatitis or hepatitis $\mathrm{C}$ virus related ACLF. In China, most ACLF patients were chronic HBV infection related. Moreover, the clinical manifestation and pathophysiology were different from alcoholic hepatitis related ACLF to HBV-ACLF. Consequently, whether these models exhibited ideal predictive value in patients with HBV-ACLF needed further investigation. It is necessary to identify an accurate prognostic scoring system based on patients with HBV-ACLF.

The ALBI grade was built to predict prognosis of 
Table 2 Independent prognostic factors for 30-day mortality by the univariate and multivariate Cox proportional hazards regression model in the derivation cohort

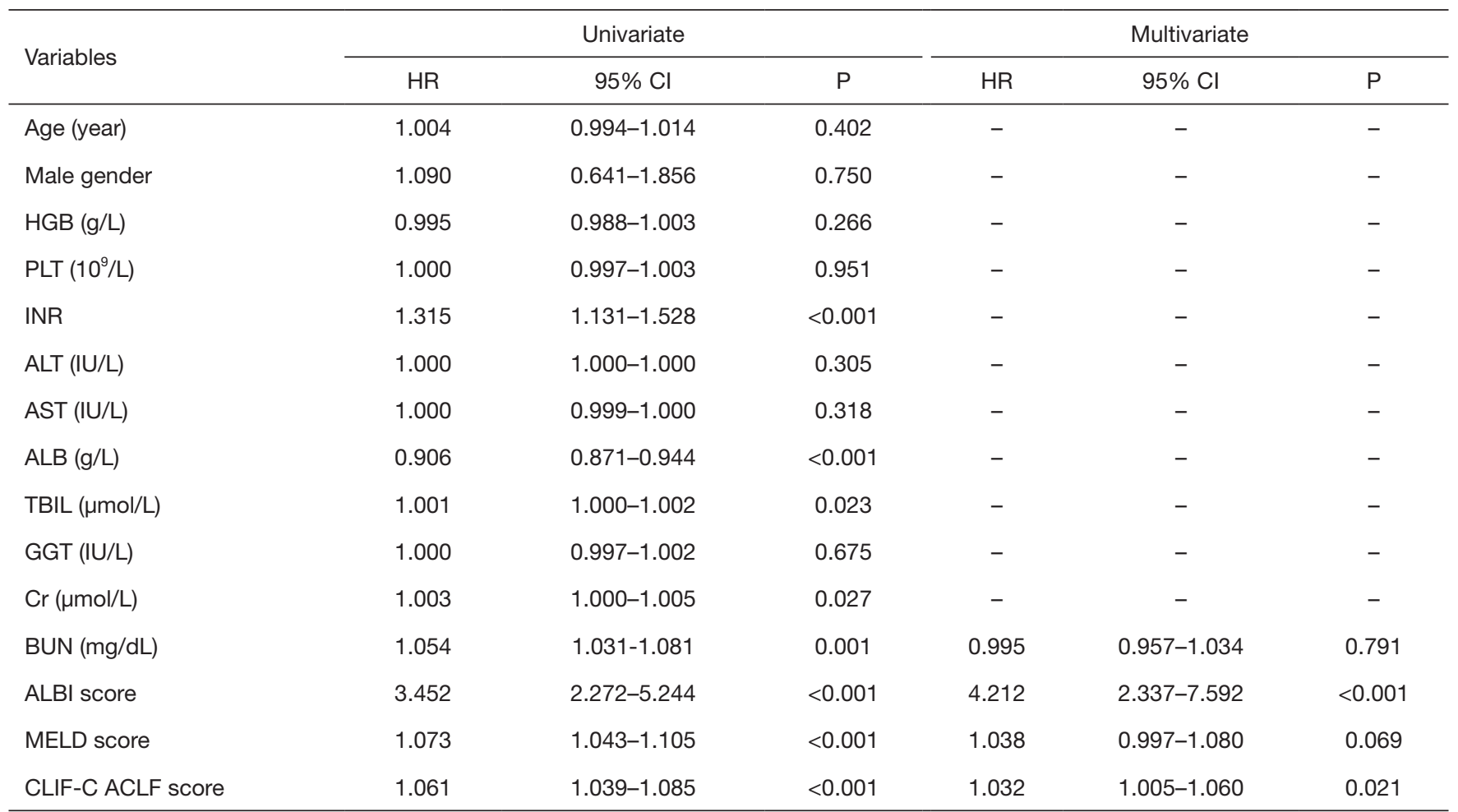

$\mathrm{HR}$, hazard ratio; $\mathrm{Cl}$, confidence interval; HGB, hemoglobin; PLT, platelet; INR, international normalized ratio; ALT, alanine aminotransferase; AST, aspartate aminotransferase; ALB, albumin; TBIL, total bilirubin; GGT, gamma glutamyl transpeptidase; Cr, creatinine; BUN, blood urea nitrogen; ALBI, albumin-bilirubin; MELD, model for end-stage liver diseases; CLIF-CACLF, CLIF-CONSORTIUM score for ACLF patients.

patients with HCC. Evidence based on 1,313 HCC patients from Japanese shown ALBI score was an accurate predictor of prognostic outcome and extended as an index of liver dysfunction (15). Another study by Chen et al. revealed that patients with lower ALBI score were associated with longer life expectancy. ALBI grade was more accurate than CP grade and MELD score for patients with HBV-related cirrhosis (31). ALBI grade was applied to predict hepatic function decline, and the conclusion shown it functioned similarly to CP score (32). Moreover, Andreatos et al. used ALBI grade to predict post hepatic liver failure in HCC patients underwent hepatectomy, and considered it as an effective predictor (33). Indeed, only serum albumin and bilirubin were involved in ALBI grade, which were entirely objective measurements that would be routinely monitored in patients with liver dysfunction. It would be easily available for ALBI grade.

A previous study enrolled 100 ACLF patients compared
CP, MELD score and ALBI score in predicting the inhospital mortality (34). It concluded that neither MELD score nor ALBI score was effective in predicting the inhospital mortality of cirrhosis patients with ACLF. However, several limitations of this study unfolded obviously. It was unreliable to make a conclusion based on insufficient data set. Moreover, the etiology of patients enrolled in the study seemed to be incomparable. And different etiology would result in different clinical manifestation, pathophysiology and prognosis. In our study, we enrolled almost $400 \mathrm{HBV}$-ACLF patients based upon strict inclusion criteria. We also evaluated clinical and biochemical parameters using univariate and multivariate Cox proportional hazard regression.

There are some limitations in the present study warrant consideration when interpreting our findings. Firstly, this is a retrospective study based on clinical data from single center, which may result in an increased possibility of selection bias and limit the generalization. Further 

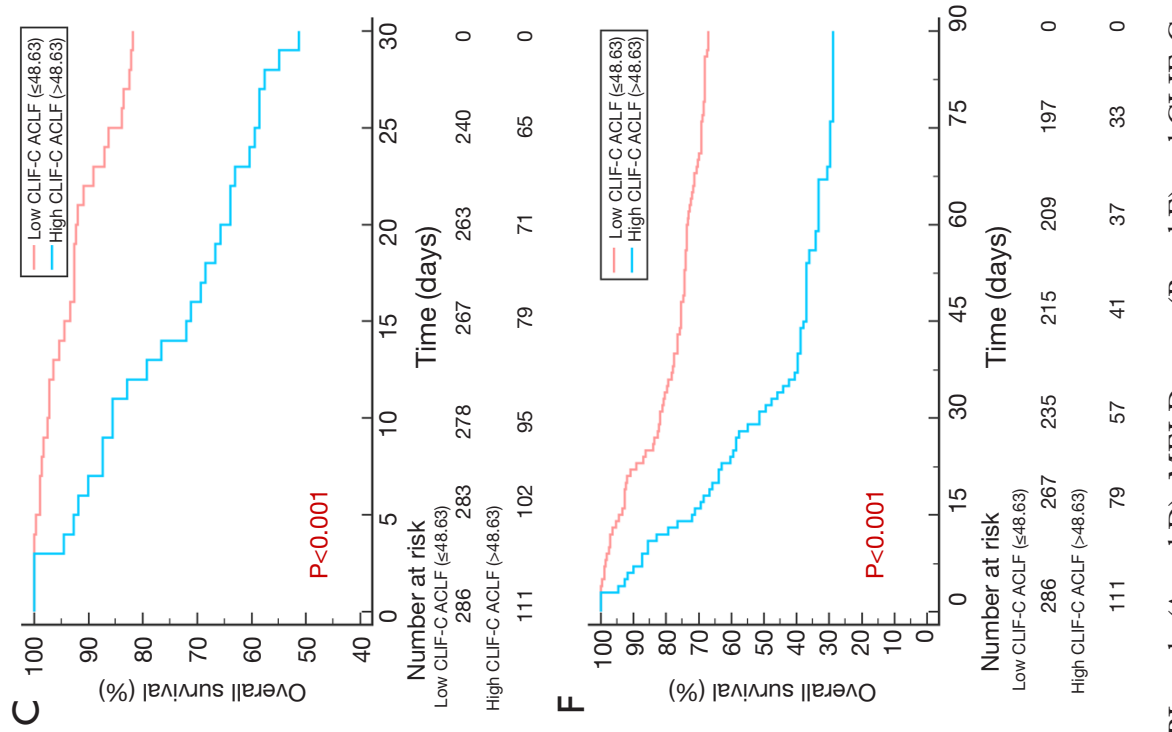

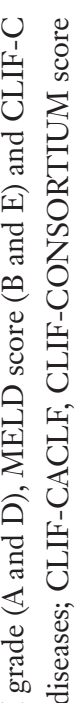

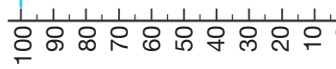

(\%) ןем!лns ॥еґәло

窎.
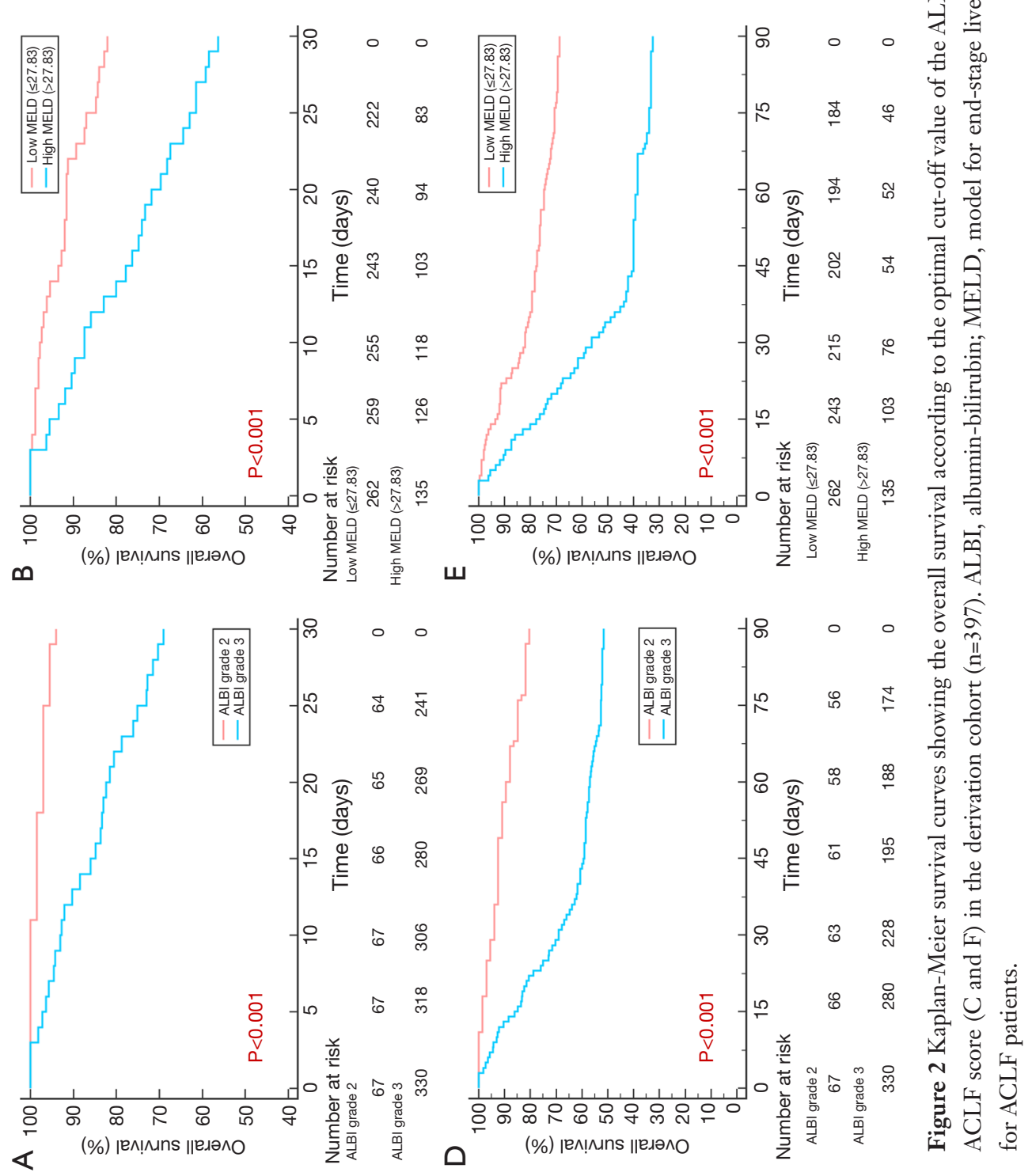
Table 3 Independent prognostic factors for 90-day mortality by the univariate and multivariate Cox proportional hazards regression model in the derivation cohort

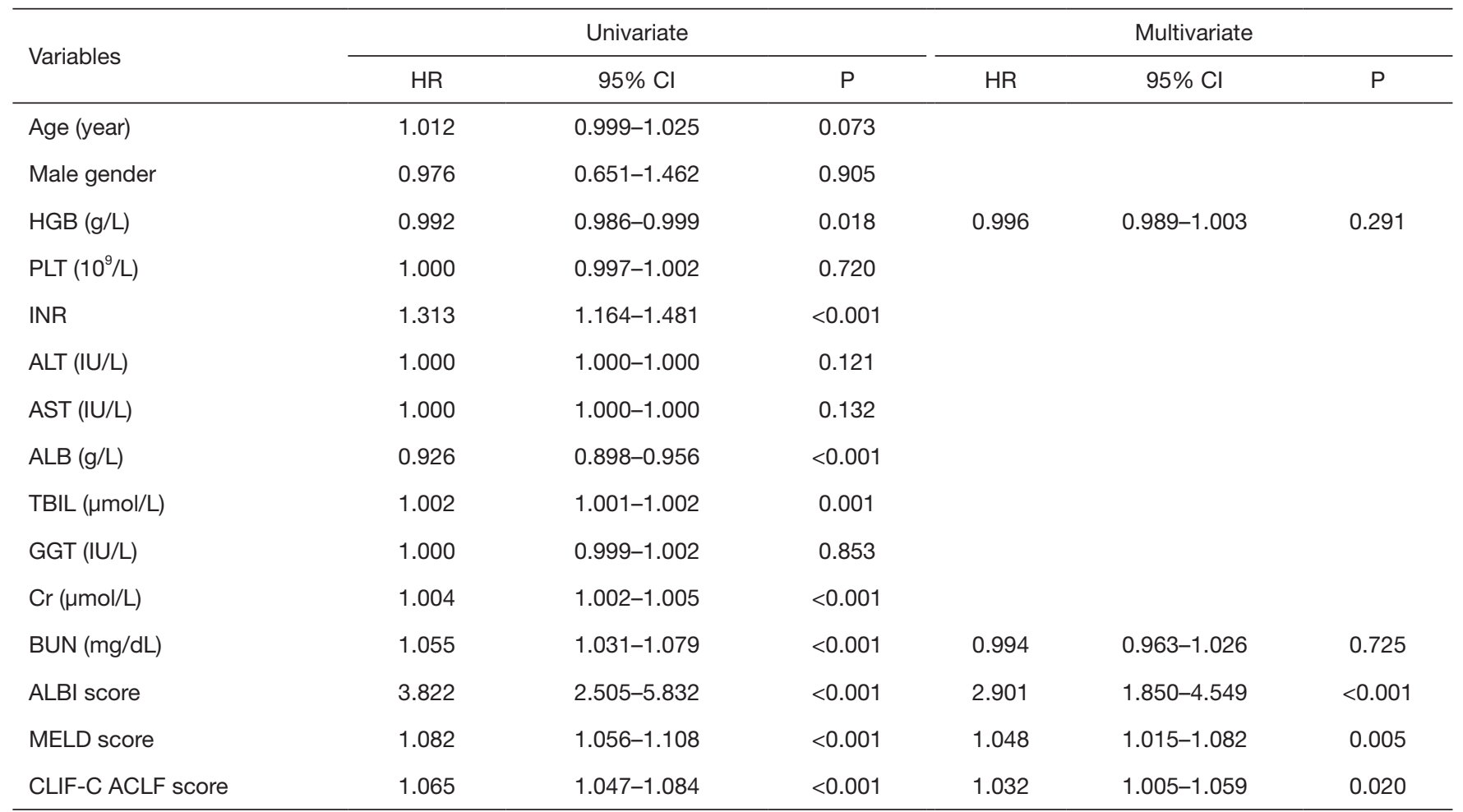

$\mathrm{HR}$, hazard ratio; Cl, confidence interval; HGB, hemoglobin; PLT, platelet; INR, international normalized ratio; ALT, alanine aminotransferase; AST, aspartate aminotransferase; ALB, albumin; TBIL, total bilirubin; GGT, gamma glutamyl transpeptidase; Cr, creatinine; BUN, blood urea nitrogen; ALBI, albumin-bilirubin; MELD, model for end-stage liver diseases; CLIF-CACLF, CLIF-CONSORTIUM score for ACLF patients.

international multi-center researches with more diversified patients should be conducted to substantiate our findings. Furthermore, all the included patients were in ALBI grade 2 or grade 3 due to the insufficient available data of ALBI grade 1 . Nevertheless, our study was carried out at an appropriate time as the lack of reliable study investigated the prognostic value of the ALBI grade that based on HBVACLF patients.
In conclusion, our study revealed that both the ALBI score, MELD score and CLIF-C ACLF score could predict 30- and 90-day mortality of HBV-ACLF accurately. Elevated ALBI score, MELD score and CLIF-C ACLF score were associated with worse prognosis. The ALBI score was associated with larger AUC than the MELD score and CLIF-C ACLF in predicting 30-day mortality. In the clinical treatment strategies decision making and 


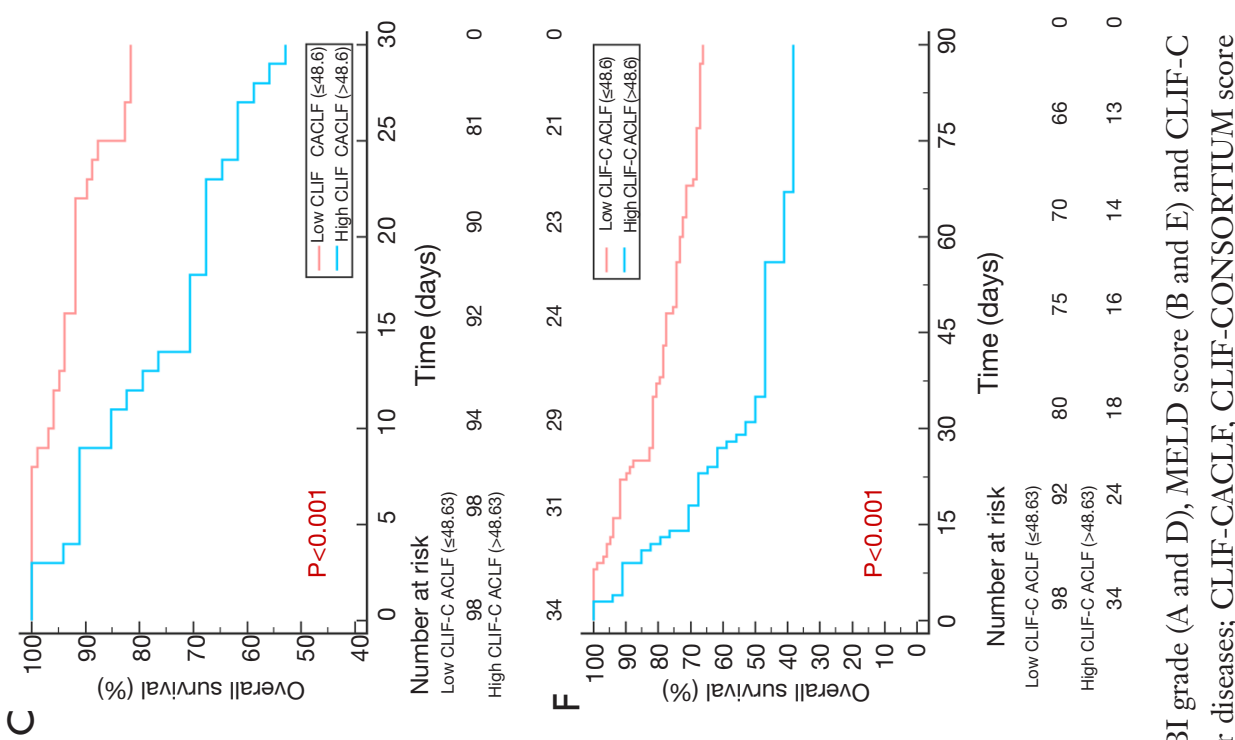

$$
\text { U }
$$
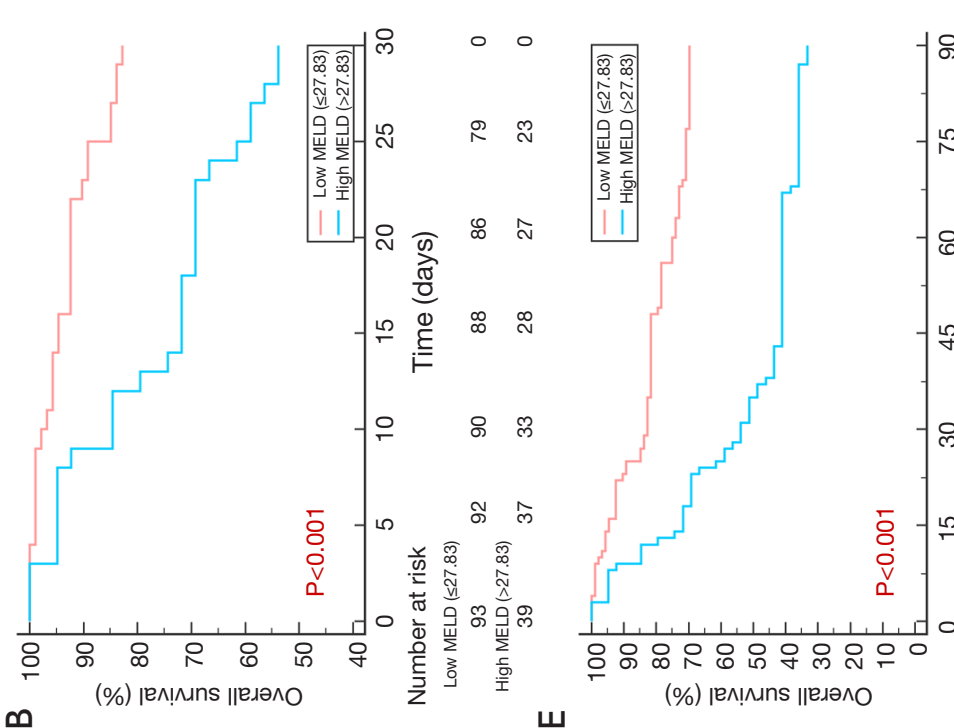

突

$\infty$

$$
\text { w }
$$

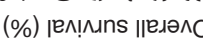

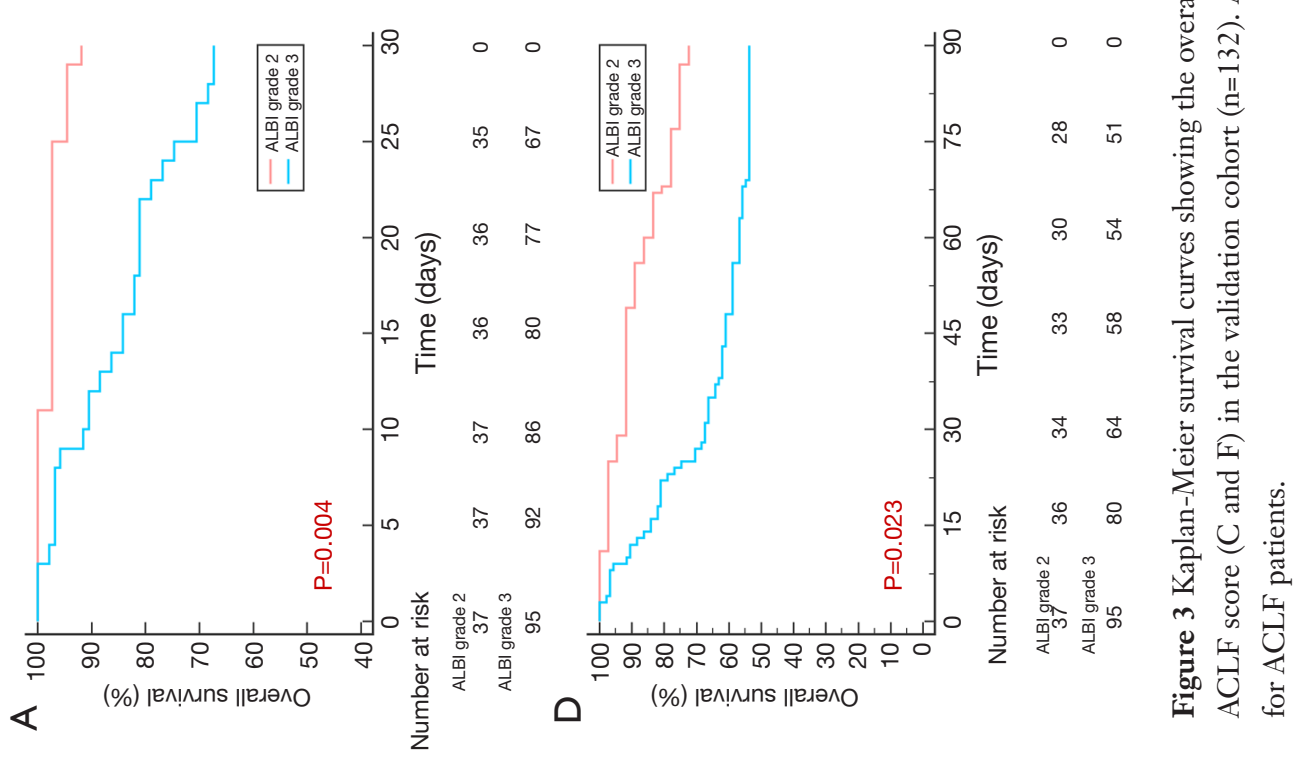


selection of appropriate candidate for liver transplantation, these three scoring systems should be routinely performed.

\section{Acknowledgments}

Funding: This work was supported by: National Natural Science Foundation of China [81570593, 81870447, 81770648, 81972286]; Science and Technology Planning Project of Guangzhou [201508020262]; Natural Science Foundation of Guangdong Province [2015A030312013]; Science and Technology Planning Project of Guangdong Province [2017A020215178].

\section{Footnote}

Reporting Checklist: The authors have completed the STROBE reporting checklist. Available at http://dx.doi. org/10.21037/apm-20-608

Data Sharing Statement: Available at http://dx.doi. org/10.21037/apm-20-608

Peer Review File: Available at http://dx.doi.org/10.21037/ apm-20-608

Conflicts of Interest: All authors have completed the ICMJE uniform disclosure form (available at http://dx.doi. org/10.21037/apm-20-608). The authors have no conflicts of interest to declare.

Ethical Statement: The authors are accountable for all aspects of the work in ensuring that questions related to the accuracy or integrity of any part of the work are appropriately investigated and resolved. This study was approved by the ethics committee of the Third Affiliated Hospital of Sun Yat-sen University (the ethical number: 2014-142), in accordance with the guidelines of the Declaration of Helsinki (as revised in 2013). Individual informed consent was waived due to the retrospective nature of the study.

Open Access Statement: This is an Open Access article distributed in accordance with the Creative Commons Attribution-NonCommercial-NoDerivs 4.0 International License (CC BY-NC-ND 4.0), which permits the noncommercial replication and distribution of the article with the strict proviso that no changes or edits are made and the original work is properly cited (including links to both the formal publication through the relevant DOI and the license). See: https://creativecommons.org/licenses/by-nc-nd/4.0/.

\section{References}

1. Lin BL, Chen JF, Qiu WH, et al. Allogeneic bone marrow-derived mesenchymal stromal cells for hepatitis B virus-related acute-on-chronic liver failure: A randomized controlled trial. Hepatology 2017;66:209-19.

2. Garg H, Kumar A, Garg V, et al. Clinical profile and predictors of mortality in patients of acute-on-chronic liver failure. Dig Liver Dis 2012;44:166-71.

3. Sarin SK, Kedarisetty CK, Abbas Z, et al. Acute-onchronic liver failure: consensus recommendations of the Asian Pacific Association for the Study of the Liver (APASL) 2014. Hepatol Int 2014;8:453-71.

4. Sarin SK, Choudhury A. Acute-on-chronic liver failure: terminology, mechanisms and management. Nat Rev Gastroenterol Hepatol 2016;13:131-49.

5. Olson JC, Kamath PS. Acute-on-chronic liver failure: what are the implications? Curr Gastroenterol Rep 2012;14:63-6.

6. Katoonizadeh A, Laleman W, Verslype C, et al. Early features of acute-on-chronic alcoholic liver failure: a prospective cohort study. Gut 2010;59:1561-9.

7. Zhang GL, Xie DY, Lin BL, et al. Imbalance of interleukin-17-producing CD4 T cells/regulatory T cells axis occurs in remission stage of patients with hepatitis $\mathrm{B}$ virus-related acute-on-chronic liver failure. J Gastroenterol Hepatol 2013;28:513-21.

8. Artru F, Louvet A, Ruiz I, et al. Liver transplantation in the most severely ill cirrhotic patients: A multicenter study in acute-on-chronic liver failure grade 3. J Hepatol 2017;67:708-15.

9. Wiesner R, Edwards E, Freeman R, et al. Model for endstage liver disease (MELD) and allocation of donor livers. Gastroenterology 2003;124:91-6.

10. Kim WR, Biggins SW, Kremers WK, et al. Hyponatremia and mortality among patients on the liver-transplant waiting list. N Engl J Med 2008;359:1018-26.

11. Jalan R, Saliba F, Pavesi M, et al. Development and validation of a prognostic score to predict mortality in patients with acute-on-chronic liver failure. J Hepatol 2014;61:1038-47.

12. Child CG, Turcotte JG. Surgery and portal hypertension. 
Major Probl Clin Surg 1964;1:1-85.

13. Pugh RN, Murray-Lyon IM, Dawson JL, et al. Transection of the oesophagus for bleeding oesophageal varices. Br J Surg 1973;60:646-9.

14. Durand F, Valla D. Assessment of the prognosis of cirrhosis: Child-Pugh versus MELD. J Hepatol 2005;42 Suppl:S100-7.

15. Johnson PJ, Berhane S, Kagebayashi C, et al. Assessment of liver function in patients with hepatocellular carcinoma: a new evidence-based approach-the ALBI grade. J Clin Oncol 2015;33:550-8.

16. Kuo YH, Wang JH, Hung CH, et al. Albumin-Bilirubin grade predicts prognosis of HCC patients with sorafenib use. J Gastroenterol Hepatol 2017;32:1975-81.

17. Liu PH, Hsu CY, Tsai YJ, et al. ALBI and PALBI grade predict survival for HCC across treatment modalities and BCLC stages in the MELD era. Hepatology 2016;63:626A.

18. Pinato DJ, Sharma R, Allara E, et al. The ALBI grade provides objective hepatic reserve estimation across each BCLC stage of hepatocellular carcinoma. J Hepatol 2017;66:338-46.

19. Kao WY, Su CW, Chiou YY, et al. Hepatocellular Carcinoma: Nomograms Based on the Albumin-Bilirubin Grade to Assess the Outcomes of Radiofrequency Ablation. Radiology 2017;285:670-80.

20. Chan AW, Chan RC, Wong GL, et al. New simple prognostic score for primary biliary cirrhosis: Albuminbilirubin score. J Gastroenterol Hepatol 2015;30:1391-6.

21. Sarin SK, Kumar A, Almeida JA, et al. Acute-on-chronic liver failure: consensus recommendations of the Asian Pacific Association for the study of the liver (APASL). Hepatol Int 2009;3:269-82.

22. Liver Failure and Artificial Liver Group, Chinese Society of Infectious Diseases, Chinese Medical Association, et al. Diagnostic and treatment guidelines for liver failure (2012 version). Zhonghua Gan Zang Bing Za Zhi 2013;21:177-83.

23. Carlson RV, Boyd KM, Webb DJ. The revision of the Declaration of Helsinki: past, present and future. Br J Clin Pharmacol 2004;57:695-713.

24. Li H, Li J, Wang J, et al. Assessment of Liver Function for Evaluation of Long-Term Outcomes of Intrahepatic Cholangiocarcinoma: A Multi-Institutional Analysis of 620 Patients. Front Oncol 2020;10:525.

25. Cai X, Chen Z, Chen J, et al. Albumin-to-Alkaline
Phosphatase Ratio as an Independent Prognostic Factor for Overall Survival of Advanced Hepatocellular Carcinoma Patients without Receiving Standard AntiCancer Therapies. J Cancer 2018;9:189-97.

26. Wan Z, Wu Y, Yi J, et al. Combining serum cystatin C with total bilirubin improves short-term mortality prediction in patients with $\mathrm{HBV}$-related acute-on-chronic liver failure. PLoS One 2015;10:e0116968.

27. Lin B, Pan CQ, Xie D, et al. Entecavir improves the outcome of acute-on-chronic liver failure due to the acute exacerbation of chronic hepatitis B. Hepatol Int 2013;7:460-7.

28. Louvet A, Labreuche J, Artru F, et al. Combining Data From Liver Disease Scoring Systems Better Predicts Outcomes of Patients With Alcoholic Hepatitis. Gastroenterology 2015;149:398-406.e8; quiz e16-7.

29. Freeman RB Jr, Wiesner RH, Harper A, et al. The new liver allocation system: moving toward evidence-based transplantation policy. Liver Transpl 2002;8:851-8.

30. Duseja A, Choudhary NS, Gupta S, et al. APACHE II score is superior to SOFA, CTP and MELD in predicting the short-term mortality in patients with acute-on-chronic liver failure (ACLF). J Dig Dis 2013;14:484-90.

31. Chen RC, Cai YJ, Wu JM, et al. Usefulness of albuminbilirubin grade for evaluation of long-term prognosis for hepatitis B-related cirrhosis. J Viral Hepat 2017;24:238-45.

32. Toesca DAS, Osmundson EC, von Eyben R, et al. Assessment of hepatic function decline after stereotactic body radiation therapy for primary liver cancer. Pract Radiat Oncol 2017;7:173-82.

33. Andreatos N, Amini N, Gani F, et al. Albumin-Bilirubin Score: Predicting Short-Term Outcomes Including Bile Leak and Post-hepatectomy Liver Failure Following Hepatic Resection. J Gastrointest Surg 2017;21:238-48.

34. Peng Y, Qi X, Tang S, et al. Child-Pugh, MELD, and ALBI scores for predicting the in-hospital mortality in cirrhotic patients with acute-on-chronic liver failure. Expert Rev Gastroenterol Hepatol 2016;10:971-80.

Cite this article as: $\mathrm{Li} \mathrm{H}$, Zheng J, Chen L, Cai J, Zhang M, Wang G. The scoring systems in predicting shortterm outcomes in patients with hepatitis B virus-related acuteon-chronic liver failure. Ann Palliat Med 2020;9(5):3048-3058. doi: 10.21037/apm-20-608 


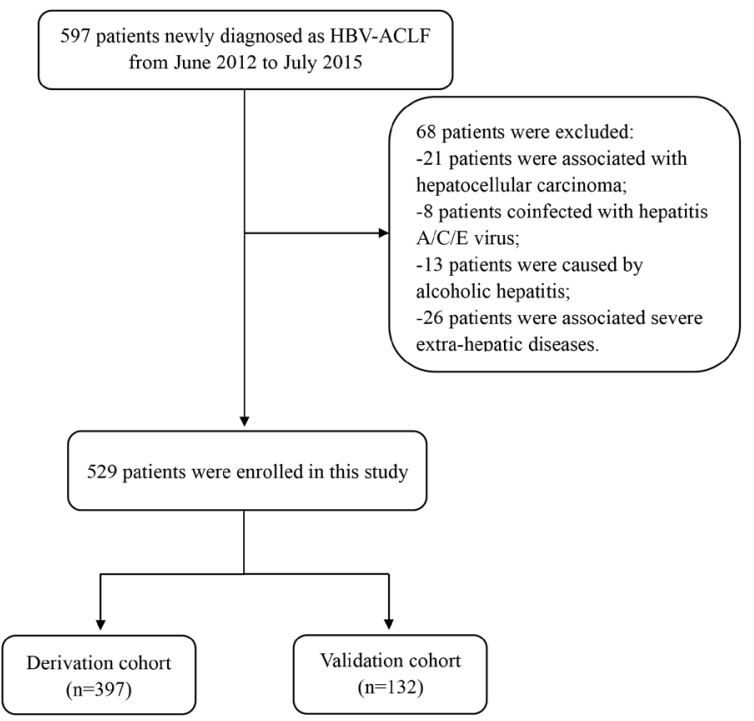

Figure S1 Flow diagram showing the procedure of selection and exclusion of patients. HBV-ACLF, hepatitis B virus related acute-onchronic liver failure. HBV-ACLF, hepatitis B virus related acute-on-chronic liver failure.

A

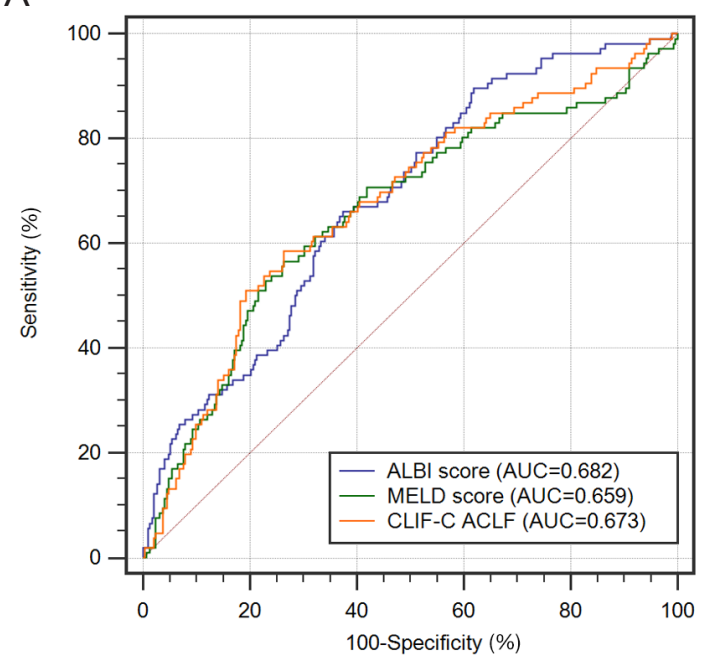

B

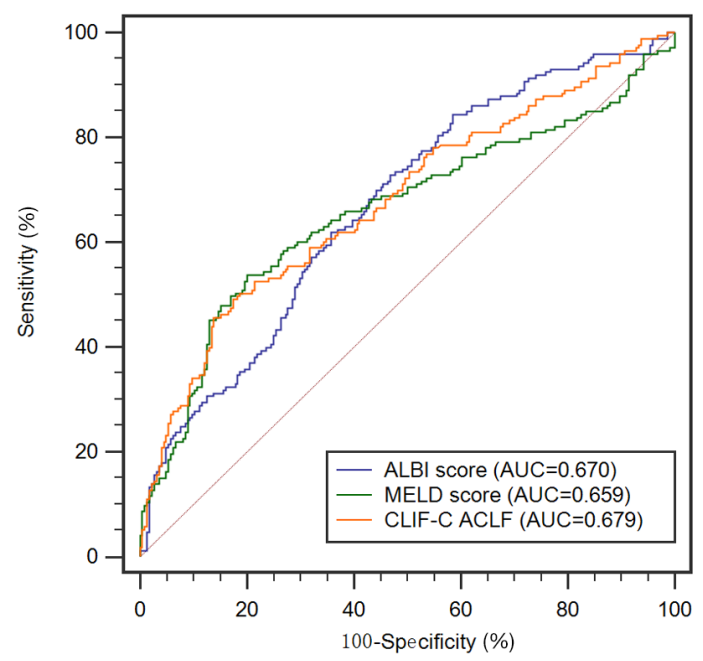

Figure S2 Comparison of area under the receiver operating characteristic (AUC) for ALBI score, MELD score and CLIF-C ACLF score in predicting 30-day mortality (A) and 90-day mortality (B). ALBI, albumin-bilirubin; MELD, model for end-stage liver disease; CLIF-C ACLF, CLIF-CONSORTIUM score for ACLF patients. 
Table S1 Diagnostic accuracy of scoring systems for prediction of 30- and 90-day mortality

\begin{tabular}{|c|c|c|c|c|c|c|c|c|}
\hline Models & AUC (95\% Cl) & Cut-off value & Sensitivity (\%) & Specificity (\%) & PPV (\%) & NPV (\%) & PLR & NLR \\
\hline ALBI score & $0.682(0.633-0.727)$ & -1.39 & 96.2 & 21.6 & 30.9 & 94 & 1.23 & 0.18 \\
\hline MELD score & $0.659(0.610-0.706)$ & 27.83 & 55.7 & 73.9 & 43.7 & 82.1 & 2.13 & 0.59 \\
\hline CLIF-CACLF score & $0.673(0.625-0.719)$ & 48.63 & 50.9 & 80.4 & 48.6 & 81.8 & 2.59 & 061 \\
\hline \multicolumn{9}{|l|}{ 90-day mortality } \\
\hline ALBI score & $0.670(0.622-0.716)$ & -1.39 & 92.5 & 24.1 & 48.5 & 80.6 & 1.22 & 0.31 \\
\hline MELD score & $0.659(0.610-0.705)$ & 27.83 & 52.6 & 80.4 & 67.4 & 68.7 & 2.68 & 0.59 \\
\hline CLIF-CACLF score & $0.679(0.630-0.724)$ & 48.63 & 45.7 & 85.7 & 71.2 & 67.1 & 3.19 & 0.63 \\
\hline
\end{tabular}

ALBI grade, albumin-bilirubin grade; MELD, model for end-stage liver disease; AUC, area under the operating characteristic; Cl, confidence interval; PPV, positive predictive value; NPV, negative predictive value; PLR, positive likelihood ratio; NLR, negative likelihood ratio.

Table S2 Independent prognostic factors for 30-day mortality by the univariate and multivariate Cox proportional hazards regression model in the validation cohort

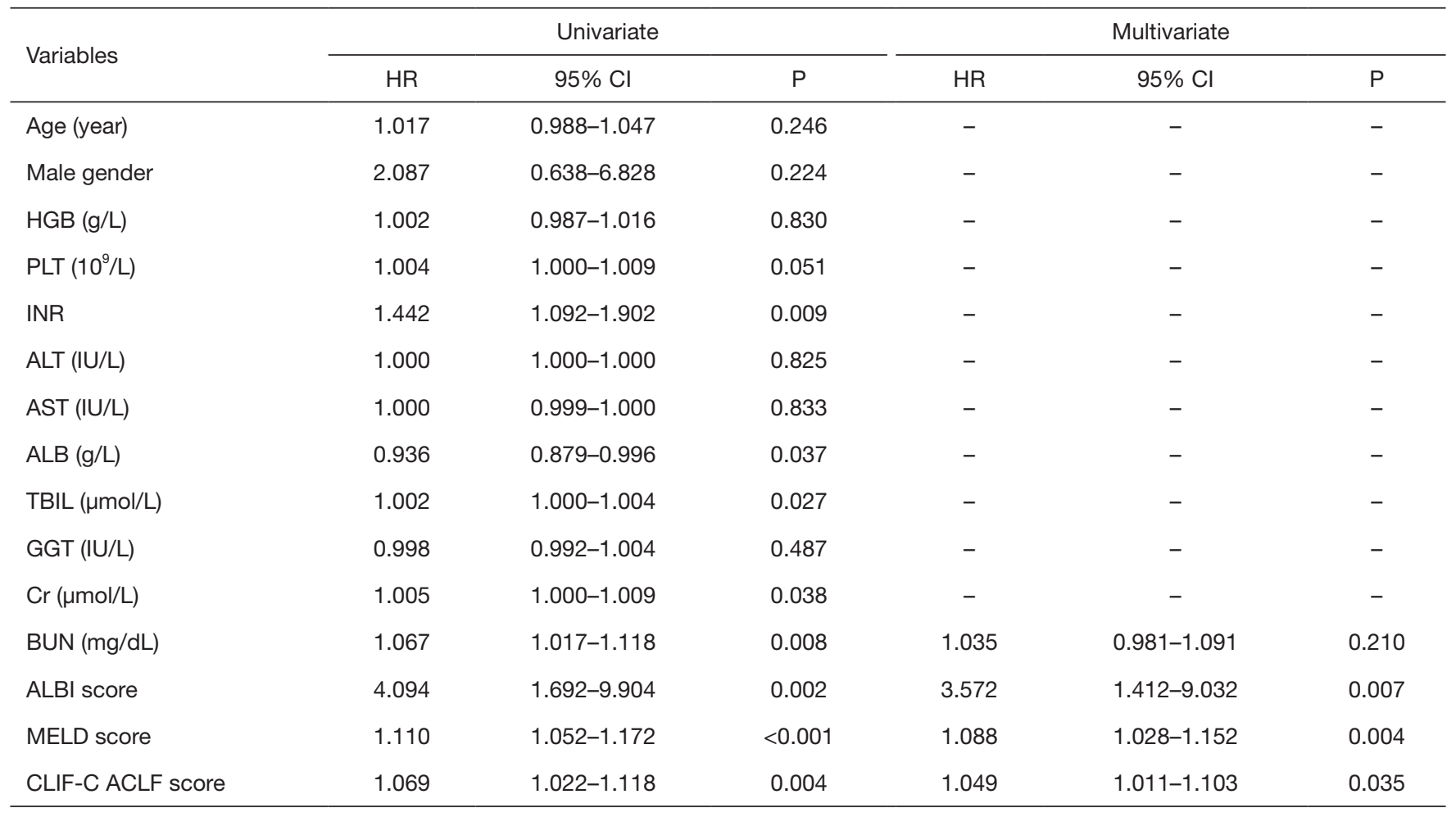

HR, hazard ratio; Cl, confidence interval; HGB, hemoglobin; PLT, platelet; INR, international normalized ratio; ALT, alanine aminotransferase; AST, aspartate aminotransferase; ALB, albumin; TBIL, total bilirubin; GGT, gamma glutamyl transpeptidase; Cr, creatinine; BUN, blood urea nitrogen; ALBI, albumin-bilirubin; MELD, model for end-stage liver diseases; CLIF-CACLF, CLIF-CONSORTIUM score for ACLF patients. 
Table S3 Independent prognostic factors for 90-day mortality by the univariate and multivariate Cox proportional hazards regression model in the validation cohort

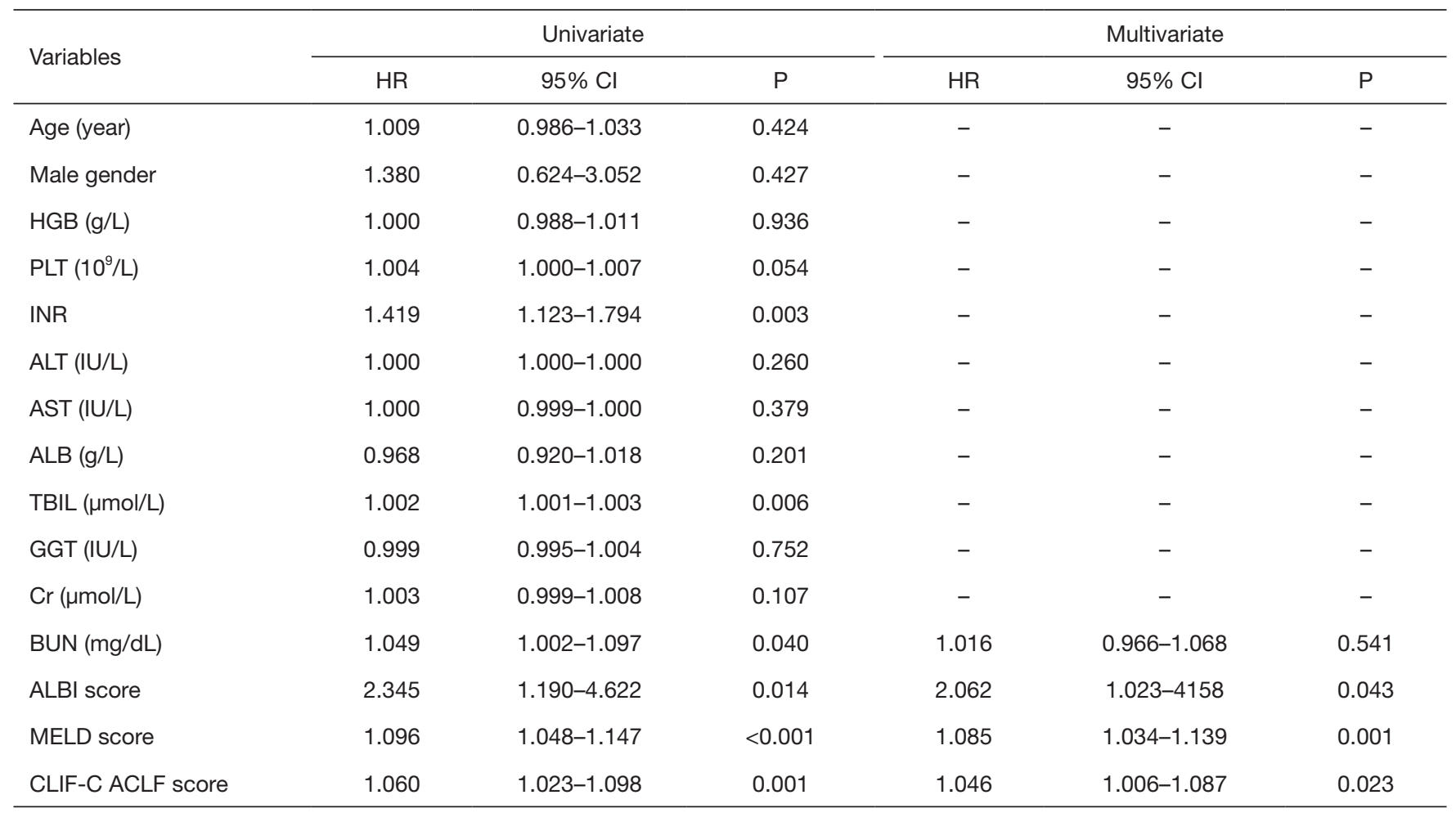

HR, hazard ratio; Cl, confidence interval; HGB, hemoglobin; PLT, platelet; INR, international normalized ratio; ALT, alanine aminotransferase; AST, aspartate aminotransferase; ALB, albumin; TBIL, total bilirubin; GGT, gamma glutamyl transpeptidase; Cr, creatinine; BUN, blood urea nitrogen; ALBI, albumin-bilirubin; MELD, model for end-stage liver diseases; CLIF-CACLF, CLIF-CONSORTIUM score for ACLF patients. 